\title{
Problemas matemáticos en el caso de un currículo: Análisis con base en el contexto y en la contextualización
}

\author{
Gilberto Chavarría-Arroyo, Universidad Nacional (Costa Rica) \\ Veronica Albanese, Universidad de Granada (España)
}

\begin{abstract}
Problemas matemáticos en el caso de un currículo: Análisis con base en el contexto y en la contextualización

\section{Resumen}

El objetivo de este estudio es analizar el contexto y la contextualización de problemas presentes en el caso del currículo de Costa Rica desarrollado en la reforma de 2012. La metodología es mixta, con un análisis cualitativo de contenido y un posterior recuento cuantitativo. El sistema de categorías se elabora a partir de la revisión bibliográfica, considerando tipos de contexto y tipos de contextualización. El 59,5\% del total de 141 problemas tienen contexto científico/matemático, muy pocos de los cuales se ajustan a una contextualización activa. Asimismo, los contextos rurales e indígenas están ausentes. De ahí que concluyamos sobre la existencia de desajustes entre los fundamentos teóricos del caso de currículo de matemáticas y los ejemplos que se plantean. Acabamos proponiendo la discusión de nuestras categorías como indicadores para construir problemas matemáticos con contextualización activa y significativa.
\end{abstract}

Palabras clave. Contexto y contextualización de problemas matemáticos; currículo matemático; fundamentos teóricos del currículo; contextualización activa y significativa.

\section{Mathematical problems in the case of a curriculum: Analysis based on context and on contextualization}

\section{Abstract}

The objective of this study is to analyze the context and contextualization of problems from the case of the Costa Rican curriculum developed in the 2012 reform. The mixed methodology consists of a qualitative content analysis and a subsequent quantitative recount. A system of categories is built from a literature review leading to the study of types of context and types of contextualization. The 59.5\% out of the total of 141 problems has a scientific/mathematics context, with contextualization of the active type in only a few of them. Moreover, contexts of the rural and indigenous types are absent. We conclude therefore that some disconnections exist between the theoretical curricular basis and the problems exemplified. Finally, we propose the further discussion of our categories as indicators for the design of mathematical problems with contextualization of the active and significant types.

Keywords. Context and contextualization of mathematical problems; mathematical curriculum; theoretical basis for the curriculum; active and significant contextualization.

\section{Introducción}

Desde hace más de seis décadas, autores como Puig (1955) indican la necesidad de presentar la matemática escolar relacionándola con la vida natural y social. Además, a partir de los años noventa del siglo pasado, surgen diversos estudios que resaltan la importancia del contexto histórico-cultural en la construcción del conocimiento matemático y que conciben las matemáticas como producto cultural, resultado de las actividades de los pueblos (Bishop, 1991; Presmeg, 2007). A pesar de ello, partimos del supuesto que la experiencia de los currículos educativos en distinto lugares del mundo sigue bastante alejada de las realidades y las culturas de los aprendices. 
Varios enfoques teóricos de la educación matemática se han interesado en la importancia del contexto de los problemas presentados en el aula. La denominada Educación Matemática Realista, por ejemplo, propone la enseñanza de matemáticas conectada con la realidad, de modo que sea cercana al aprendiz y relevante para la sociedad (Freudenthal, 2002); así, la matemática se concibe como actividad humana de reconocimiento y resolución de problemas del entorno. La denominada Enseñanza Culturalmente Relevante pone el foco en la experiencia y cultura del aprendiz; esto implica partir de situaciones culturales y de relevancia social para el desarrollo de pensamiento crítico (Gutstein, Lipman, Hernandez, \& De los Reyes, 1997).

Para el denominado Programa Etnomatemática (D’Ambrosio, 2008), múltiples prácticas matemáticas emergen de las actividades cotidianas que están impregnadas por el contexto y la cultura. Gracias a este Programa, han surgido ayudas instruccionales, materiales y la posibilidad de relacionar contenidos y confeccionar currículos que contemplen el contexto y los valores culturales de las matemáticas (Bishop, 2005). Fuentes (2013) indica que las etnomatemáticas pueden ser llevadas al aula y aportar al profesorado en la planificación, ejecución y evaluación de su práctica docente, al considerar, en las actividades de mediación, que el mundo social y la cultura en la cual vive el aprendiz son cruciales para su formación. Nótese que indicamos con Etnomatemática, mayúscula y singular, el programa de investigación, y con etnomatemáticas, minúscula y plural, las diversas matemáticas culturales que son objeto de estudio del Programa. En la misma línea, Peña (2014) considera que en la educación formal es necesario contextualizar las matemáticas, ya que:

... las matemáticas desconectadas de la historia, de otros conocimientos, y del entorno, de manera natural nos han llevado a ignorar los conocimientos matemáticos de los estudiantes, lo que ha tenido implicancias pedagógicas para el desarrollo potencial del pensamiento matemático de estudiantes de aulas culturalmente homogéneas o diversas. (p. 175)

Por la importancia que otorgamos al contexto local, enmarcamos nuestra investigación en la Etnomatemática, sin olvidar aportes de otros enfoques referentes a las nociones de contexto y de contextualización. Se trata de una investigación en curso en el marco de la investigación de tesis doctoral del primer autor.

Costa Rica, país cuyo currículo es nuestro objeto de investigación, se ha mostrado atenta a estos cambios educativos. En 2012 puso en práctica una reforma con la implantación de un nuevo currículo de matemática que se materializa en el documento de los Programas de Estudio de Matemáticas (MEP, 2012), en el que se da énfasis a la resolución de problemas ligados al entorno físico, social y cultural del aprendiz. Para el director de esta reforma, la contextualización de las matemáticas juega un papel determinante (Ruiz, 2000); en su análisis de los primeros años de implementación de la reforma, insiste en el papel relevante de los contextos reales en la enseñanza de matemáticas, indicando que se deben propiciar "experiencias cercanas a la vida real y cotidiana" (Ruiz, 2013, p. 53). Algo más tarde, el interés internacional por esa reforma del currículo queda probado al caracterizarse, en Planas (2015), como una perspectiva de la praxis en educación matemática que debe ser examinada en profundidad.

Los Programas de Estudio de Matemáticas son un documento de 518 páginas, que se proporciona a cada docente, tanto de primaria como de secundaria, y abarca desde primero hasta undécimo grado. El documento contiene información relativa a cada unidad temática (relaciones y álgebra, estadística y probabilidad, números, medidas y geometría), estructurada según tres elementos: conocimientos, habilidades específicas e indicaciones puntuales. Estas últimas tienen la finalidad de proporcionar al profesorado 
ejemplos concretos de tareas y problemas para presentar en el aula y son un material de consulta cercano para el profesorado del país.

En nuestro trabajo, analizamos el contexto y la contextualización de los problemas matemáticos presentes en las indicaciones puntuales de los Programas de Estudio de Costa Rica para el III y IV ciclo (de séptimo a undécimo grado). En particular, analizamos la conexión entre los fundamentos teóricos sobre contextualización activa que expone el MEP (2012) y los ejemplos propuestos en las indicaciones puntuales. Es decir, examinamos si lo que se declara en los fundamentos del currículo se aplica en los ejemplos propuestos. A la vez, abordamos la presencia de problemas con contextualización significativa, basados en la perspectiva etnomatemática expuesta en Albanese, Adamuz-Povedano y Bracho-López (2017).

\section{Problemas matemáticos, contexto y contextualización}

A diferencia de los ejercicios, los problemas matemáticos requieren alta demanda cognitiva al resolutor ya que no cuenta con un procedimiento algorítmico que conduzca de manera directa a la solución (Blanco y Pino, 2016). El Programa de Estudios de Matemática del MEP (2012) no profundiza en la diferenciación entre ejercicio y problema, por lo que nosotros nos centramos en el análisis de los problemas asumiendo que efectivamente pueden considerarse así.

La relación de las matemáticas con el contexto es intrínseca al desarrollo histórico del conocimiento matemático que se ha ido construyendo en cada sociedad y cultura para responder a las situaciones problemáticas emergentes (D’Ambrosio, 2008). Niss (1995) señala la importancia de las matemáticas en la sociedad según sus aplicaciones a: (1) la ciencia aplicada, al ser las matemáticas fundamento de áreas científicas como la física, ingeniería, biología y economía, cada una de ellas a su vez con aplicaciones e implicaciones socialmente trascendentes; (2) las prácticas especializadas, mediante las cuales se realizan toma de decisiones y predicciones para fenómenos sociales, económicos y naturales; y (3) las prácticas no especializadas de la vida cotidiana. Al respecto y considerando que la educación está enmarcada en un contexto social, las matemáticas no están desligadas de valores, intereses, ni de circunstancias ideológicas, políticas, económicas y culturales. Sobre la noción de contexto en educación matemática y de acuerdo con Niss (1995), Planas y Alsina (2009) sugieren que se tenga especialmente en cuenta la situación generatriz de preguntas o problemas que requieren de la activación de conocimientos y prácticas matemáticas. Así, el contexto engloba las situaciones con sentido para el aprendiz y fomenta su pensamiento matemático crítico, en línea con Gutstein et al. (1997).

Otra aproximación a la noción de contexto es la de Ramos y Font (2006), quienes la analizan diferenciando dos tipos en cuanto a matemáticas se refiere. Por una parte, el contexto "como un ejemplo particular de un objeto matemático" (p. 532) y, por otra, el contexto que permite enmarcar dicho objeto en el entorno. De ahí, el contex to puede ser 1) una entidad meramente matemática, por ejemplo, el contexto de espacios vectoriales, o 2) la situación del ambiente donde se desarrolla un contenido en particular. Esa segunda concepción de contexto (que es la que tomamos en este estudio), se indica como uso ecológico, ya que el contenido matemático tendrá un significado o uso distinto, según lugar, grupo social o temporalidad de referencia.

Con matices ligeramente distintos, Nuñez y Font (1995) se refieren a la contextualización en la educación matemática como "trabajar los conceptos en diferentes contextos concretos a fin de conseguir, por una parte, su significatividad y 
funcionalidad y, por otra, facilitar los procesos de abstracción y generalización" (p. 293). Así, la contextualización de un problema matemático está intrínsicamente relacionada con el uso ecológico del contexto en el que este se enmarca.

\subsection{Relevancia del contexto}

Hay diversas clasificaciones respecto a los contextos en problemas de matemáticas. Revisamos las que tomamos en cuenta para generar el sistema de categorías. Rico (2006) analiza la contextualización de los problemas matemáticos en el denominado análisis de la fenomenología, como parte del análisis de contenido. Basándose en la propuesta de la OECD (2004) y en relación con lo planteado por Niss (1995), clasifica los contextos de problemas según la situación en la que se enmarcan:

Personales. Referidos a actividades diarias del aprendiz (prácticas no especializadas)

Educativos y ocupacionales. Referidos a un centro escolar o entorno de trabajo (prácticas especializadas y no especializadas)

Públicos. Referidos a la comunidad y a eventos importantes de la vida pública (prácticas especializadas)

Científicos. Referidos a la comprensión de un proceso tecnológico, una interpretación teórica o un problema matemático (ciencia aplicada)

En esta investigación, además de los tipos anteriores, consideramos otras categorías para los contextos de los problemas matemáticos. En primer lugar, tomamos 'contexto costarricense' dado que los Programas de Estudio de Matemáticas del MEP (2012) enfatizan que el currículo debe responder a la realidad nacional. Refiriéndose a Costa Rica, se aclara que "la utilización de problemas como generadores de la organización de las lecciones ofrece oportunidades valiosas para conectar con las necesidades de nuestro país" (p. 19). Se incentiva así la presentación de problemas que provengan del entorno y de la idiosincrasia costarricenses. En segundo lugar, tomamos 'contexto rural' y 'contexto urbano'. Aroca (2003) expresa la necesidad de considerar las matemáticas que provienen tanto de las zonas rurales como urbanas de los países:

Muchas de las etnomatemáticas rurales y urbanas son parte esencial de la reserva cultural de un país por ser únicas, por esa acumulación histórica, por la región donde se desarrollan, por el tipo de valores que conservan, por los sistemas culturales que le dan sentido, por su forma tradicional de transmitirse que involucra uno o todos los sentidos. (p. 116)

Este investigador remarca la importancia de llevar a las aulas los contextos, tanto urbanos como rurales, donde se desarrollan las matemáticas. Nosotros consideramos contexto urbano al relacionado con la urbe o ciudad; contexto rural al que involucra la vida de campo; y añadimos 'contexto indígena', que se refiere al que contempla aspectos de la vida de algún grupo originario del país, dado que suelen presentar características distintas respecto a los contextos rurales no indígenas.

Para el momento del estudio, según el informe más reciente del Instituto Nacional de Estadística y Censo (2012), la población urbana en Costa Rica es de 72,8\% y por ende la rural de 27,2\%. La población indígena corresponde al 2,4\% de los habitantes, esto es 104.143 indígenas, donde alrededor del $80 \%$ habitan zonas rurales.

\subsection{Relevancia de la contextualización}

Los Programas de Estudio de Matemáticas del MEP (2012) explican que la contextualización fortalece el papel activo del aprendiz, comprometiéndolo con su 
aprendizaje, mediante el uso y diseño de modelos matemáticos. Se establece una diferencia entre lo que se trabajaba en currículos pasados como contextualización, y lo que ahora se denomina contextualización activa. En contraposición a los problemas matemáticos que se "decoran" con elementos cotidianos que son prescindibles, esta nueva alternativa propone que los problemas matemáticos presenten contextos necesarios para ser resueltos:

Diseñar problemas sacados de las informaciones de prensa, de la escuela, de la comunidad, de la clase, de Internet. Los mismos "problemas" tradicionales que aparecen en muchos libros de texto (como apéndice) pueden ser enriquecidos si se colocan en la perspectiva de la modelización y usados para construir capacidades cognitivas superiores (p. 96).

Para Ruiz (2017), un problema es de contextualización artificial cuando el contexto que se brinda no es necesario para su resolución. Este autor concibe estos contextos y la contextualización a la que dan lugar como adornos y propone no utilizarlos. Freudenthal (2002) ya explicó de modo similar que enfocar el contexto como "ruido" perturba la claridad del mensaje matemático.

En MEP (2012), se sostiene que la contextualización activa es posible con la modelización y mediante la manipulación de entornos reales, acorde a cada nivel educativo. A la vez, se sugiere el uso de la historia para realizar conexiones de manera natural y realista, considerando no solo los aportes de las llamadas culturas occidentales, sino también los producidos en América.

En el análisis de Ruiz (2017) sobre la puesta en marcha de los Programas de Estudio de Matemáticas y relacionado con la contextualización de los problemas, se escribe: "las situaciones [...] deben tener significado y no ser artificiales, [evitando] situaciones matemáticas abstractas disfrazadas por medio de un contexto real" (pp. 72-73). Para evaluar si un problema está bien contextualizado (lo que se interpreta como equivalente a la denominada contextualización activa), Ruiz plantea que:

[L]as situaciones de contexto real deben estar relacionadas con la tarea matemática que se propone de una forma precisa .... ¿Es necesario el contexto para realizar la tarea? Si la tarea planteada no resuelve los desafíos del contexto o se podría prescindir del mismo para realizarla o sus resultados no aportan algo significativo para el contexto, no se logra lo que se persigue con el eje disciplinar. La clave debe ser la búsqueda de un involucramiento intelectual del estudiante por medio de situaciones que le permitirán desarrollar sus habilidades y capacidades matemáticas. (p. 74)

Nosotros entendemos por problema matemático con contextualización activa, aquel en el cual el contexto es imprescindible para resolver el problema, es decir que proporciona sentido a los conceptos y contenidos matemáticos implicados en la resolución y es realístico o imaginable. En otras palabras, el problema en el que el contexto es necesario para entender su formulación y plantear su resolución.

\subsection{Contextualización significativa}

D’Ambrosio (2008) sostiene que “contextualizar la matemática es esencial para todos" (p. 92). La Etnomatemática como programa de investigación permite "entender el saber/hacer matemático a lo largo de la historia de la humanidad, contextualizado en diferentes grupos de interés, comunidades, pueblos y naciones" (p. 17). Si las matemáticas se desarrollan en la cultura de un grupo social determinado, su estudio debe realizarse a partir de la realidad del grupo donde se lleva a cabo el proceso de enseñanza y aprendizaje. En esta línea, un problema matemático contextualizado, desde la Etnomatemática, es aquel que responde a una situación que se presenta en la cultura o 
realidad cercana al aprendiz. En otras palabras, el planteamiento del problema es de alguna forma necesario para la realidad en la que se enmarca.

La noción de contextualización significativa se fundamenta también en la noción de autenticidad de las tareas (Palm, 2008). Una tarea auténtica es aquella que parte de: 1) un evento que ha ocurrido o tiene alta probabilidad de ocurrir, 2) una pregunta que concuerda con un contexto ajeno a la escuela, 3) de información y datos que son realísticos o imaginables. En Albanese et al. (2017) desde una perspectiva etnomatemática, la contextualización de las tareas se torna significativa cuando la situación creada para el problema es análoga a una que efectivamente pueda surgir en un contexto real (lo que coincide con nuestra interpretación del criterio de autenticidad), y los conceptos y procedimientos matemáticos se ponen en práctica de forma similar a cómo lo harían las personas que se enfrentan a ese problema en la realidad.

Nosotros consideramos un problema con contextualización significativa como aquel que surge de un contexto real, donde los procedimientos y conceptos matemáticos se ponen en práctica de forma similar a como lo haría el grupo que se enfrenta al problema en la realidad. Esta definición va algo más allá de la autenticidad ya que el planteamiento del problema y su resolución deben tener significado para la realidad sugerida. Según Freudenthal (2002), cuando los contextos son familiares y cercanos para el aprendiz, son puntos de partida de su práctica matemática, con lo que se promueve su sentido común y sus estrategias cognitivas, hasta avanzar hacia niveles de mayor formalización.

\section{Metodología y métodos}

Nuestra metodología es mixta, partiendo de un análisis cualitativo del contenido con apoyo en métodos cuantitativos de síntesis de datos. Con respecto al análisis de contenido, como Bardin (2012) lo entendemos a modo de "conjunto de técnicas de análisis de comunicaciones" (p. 23), que lleva a obtener indicadores mediante procedimientos sistemáticos. El documento analizado se titula Programas de Estudio de Matemáticas del MEP (2012) y se focaliza en las indicaciones puntuales.

Cabrera (2005) expone, dentro del análisis de contenido, la importancia de establecer categorías, señalando que es "uno de los elementos básicos para tener en cuenta en la elaboración y distinción de tópicos a partir de los que se recoge y organiza la información" (p. 64). Por tal razón, sintetizamos los constructos más relevantes, emanados del estudio bibliográfico, a fin de elaborar una serie de categorías deductivas que dieron origen a subcategorías para el análisis de cada problema.

El sistema de dos categorías (contexto y contextualización) y subcategorías se fue ajustando durante el análisis con algunas eliminaciones por no disponer de información suficiente en las indicaciones puntuales. Presentamos las categorías y subcategorías que se utilizaron, indicando los documentos de los cuales se extrajeron:

- Contextos: personal, educativo/ocupacional, público, científico (Rico, 2006; OECD, 2004; costarricense (MEP, 2012); urbano, rural, indígena (ampliado de Aroca, 2013).

- Contextualizaciones: activa, artificial (Ruiz, 2017; MEP, 2012); significativa y no significativa (Palm, 2008; Albanese et al., 2017).

Analizamos los 141 problemas en las indicaciones puntuales del Programa de Estudio de Matemáticas del MEP (2012) del III y IV ciclo de la educación formal (edades entre 12 y 17 años), con el fin de ubicarlos en subcategorías. 35 son del área de 
números, 40 de geometría, 35 de relaciones y álgebra y 31 de estadística y probabilidad, según el propio documento.

Dentro de la categoría 'contexto', tenemos las subcategorías 'personal', 'educativo/ocupacional', 'pública' y 'científica', además de las subcategorías 'urbano', 'rural' e 'indígena', y finalmente la 'costarricense'. Para los problemas de contexto personal, educativo/ocupacional y público (se excluyen los de contexto científico, ya que, por su definición, no están ligados a un contexto cercano a la vida cotidiana), consideramos la categoría 'contextualización', analizando si es 'activa' o 'artificial'. Para ello, planteamos el siguiente interrogante: ¿es necesario el contexto que se proporciona para resolver el problema? Si la respuesta es positiva, se concluye que el problema es de contextualización activa; de lo contrario, se concluye que es de contextualización artificial. A su vez, los problemas con contextualización activa se clasifican según la subcategoría 'significativa' o 'no significativa'. Aquí nos preguntamos: ¿el problema se encuentra como tal en la vida real? ¿la pregunta del problema refleja una situación en la realidad? De ser positivas las respuestas, se concluye que el problema es de contextualización significativa.

La definición de las categorías fue avalada por el juicio de dos expertas involucradas en el Programa de Etnomatemática (María Elena Gavarrete y Natividad AdamuzPovedano) a las que se preguntó sobre el sistema de subcategorías. Gracias a sus aportes: a) se creó una subcategoría que inicialmente no estaba contemplada, la subcategoría 'costarricense' para contexto; b) se eliminó la subcategoría 'simulado' para contexto debido a la poca información en los problemas analizados para abordar ese aspecto; c) se precisó la definición de la subcategoría 'significativa' para contextualización asociándola a la noción de tarea auténtica.

\section{Resultados}

Seguidamente presentamos resultados del análisis de contenido y del recuento cuantitativo de los problemas propuestos en las indicaciones puntuales del Programa de Estudios de Matemáticas (MEP, 2012) según nuestras categorías y subcategorías. Aportamos ejemplos sobre cómo se han llevado a cabo los análisis.

\subsection{Resultados sobre los contextos de los problemas}

Con respecto a la clasificación dada en PISA (OECD, 2004), de las indicaciones puntuales de los Programas de Estudio de Matemáticas en Costa Rica, el contexto científico es el más presente con un $59,5 \%$ de los problemas. Dentro del contexto científico destacan dos tipos: los que corresponden a la aplicación de alguna fórmula y los que desarrollan un conocimiento matemático dentro de la disciplina. En el primer caso, la mayoría de estos problemas permiten enlazar las matemáticas con otras áreas del saber, tales como física, química, biología o finanzas. La Figura 1 representa los valores absolutos de la clasificación de los problemas analizados.

Un ejemplo de problema con contexto científico se presenta en el área de relaciones y álgebra: "La temperatura en grados Fahrenheit es función de la temperatura en grados Celsius y está modelada por la ecuación $F(C)=9 / 5 C+32$. Expresar $C$ como función de F” (MEP, 2012, p. 413). Aquí se nota conexión con la física. Un segundo ejemplo se relaciona con el teorema de Pitágoras, donde se integran conocimientos tales como distancia entre puntos y clasificación de triángulos según medidas de ángulos y lados: "Dadas las siguientes coordenadas de los vértices de un triángulo $\mathrm{A}(2,1), \mathrm{B}(6,5)$ y 
$\mathrm{C}(9,2)$, clasifique el triángulo de acuerdo con la medida de sus ángulos y la medida de sus lados" (MEP, 2012, p 317).

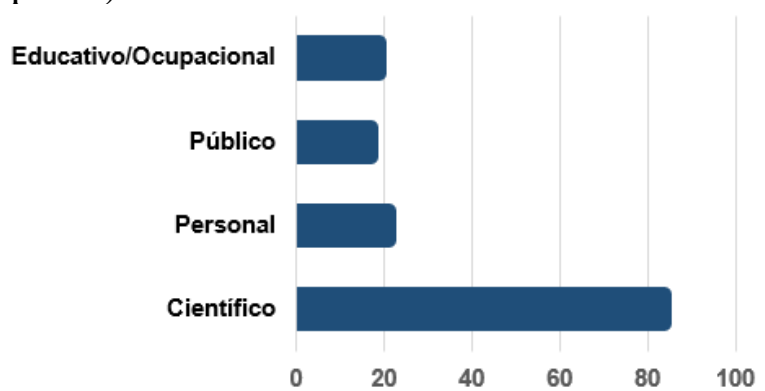

Figura 1. Distribución absoluta del tipo de contexto según PISA

Dentro de los contextos personales, se presentan problemas de compras, préstamos, actividades recreativas y recetas, especialmente en el área de números. Por otra parte, los contextos públicos están más presentes en el área de estadística y probabilidad, donde destacan ejemplos que fomentan el análisis, la toma de decisiones y propician la discusión de temas cívicos, de salud y problemáticas actuales. Con respecto a los contextos ocupacionales, los problemas corresponden al cálculo de precios, ganancias y situaciones que se pueden presentar en algunos oficios. En el contexto educativo los problemas son mayormente de estadística y probabilidad.

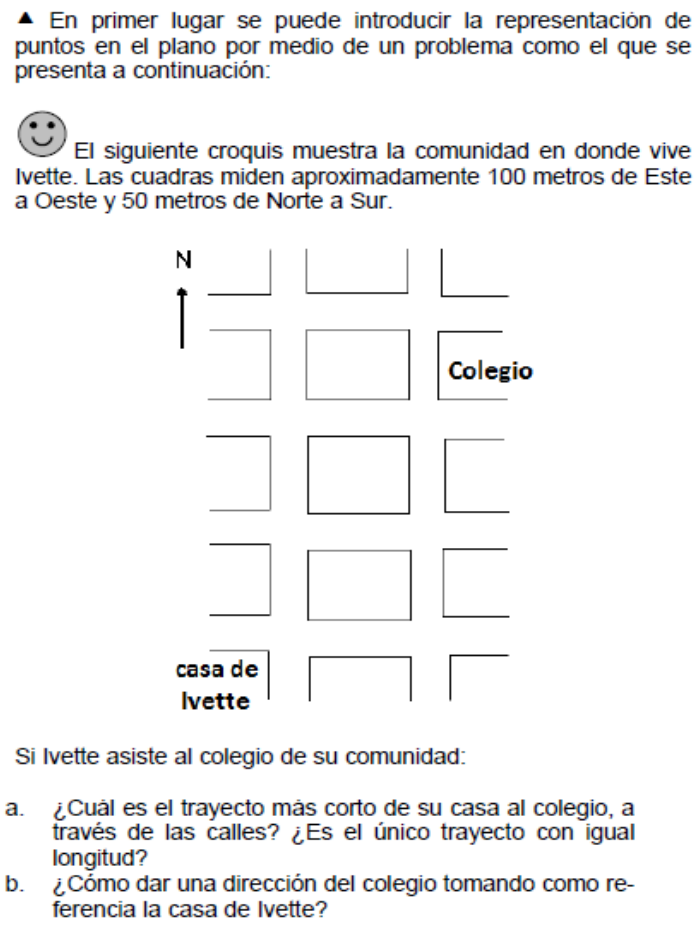

Figura 2. Problema con contexto público y urbano. Fuente: MEP (2012, p. 306)

De los 141 problemas, 22 han sido clasificados según si el contexto es urbano, rural o indígena. En otros, a pesar de que se plantea un entorno cercano al aprendiz como, por ejemplo, los juegos de azar, no ha sido posible establecer una zona geográfica. De esos 22 problemas, ninguno corresponde a un entorno rural o indígena. La realidad sugerida en los ejemplos del MEP responde siempre a contextos urbanos. Estas indicaciones puntuales pueden ser distantes a la realidad que se vive en zonas no urbanas. En las zonas rurales no es común una distribución de calles y avenidas, o el uso de "cuadras" para nombrar 100 metros de distancia, por lo que un problema como el de la Figura 2, 
no es cercano para un aprendiz que vive en el campo. Incluso, en la mayoría de zonas urbanas, es difícil que exista una distribución vial y de viviendas como la del croquis.

Otro ejemplo es el de un problema que se sitúa en una "feria del agricultor" (mercado de verduras, frutas y hortalizas generalmente en las calles durante los fines de semana), donde se presenta una lista de productos en venta, con sus respectivos precios y se solicita la cantidad de dinero que se gasta en ciertas compras (MEP, 2012, p 276). Esto es bastante cercano para algunos habitantes de la zona urbana, que desde pequeños van con sus familias a comprar verduras y frutas y hacen cálculos para verificar que les alcance el dinero. Pero no es cercano para habitantes de zonas indígenas, donde el uso del dinero es casi inexistente y se sigue utilizando el trueque, siendo este un elemento diferencial entre realidades indígenas y rurales no indígenas.

De la totalidad de problemas, $26(18,43 \%)$ tienen información para ser categorizados conforme a la realidad de Costa Rica. De ellos, 21 mencionan en cierta forma a Costa Rica. Por otra parte, un grupo de problemas se caracteriza por proporcionar datos de instituciones locales tales como la Refinadora Costarricense de Petróleo, la Caja Costarricense de Seguro Social o el Consejo Nacional de Producción. También se mencionan actividades recreativas, servicios públicos o datos demográficos. En cuatro problemas (de los 21 referidos a Costa Rica), si bien se hace alusión al país, la situación descrita no corresponde a la realidad costarricense. Por ejemplo, en el siguiente problema se menciona Costa Rica, pero para la resolución se debe suponer que las carreteras son rectas, lo cual no sucede en este país.

Carolina sale de su casa y se dirige al hogar de su mamá que se ubica $2 \mathrm{~km}$ al Sur del suyo. Luego de saludarla y conversar con ella, le informan que su hermano Andrés (quien estudia en el extranjero y llevaba más de 5 años de no visitar a su familia) llegó a Costa Rica y que se encuentra en su casa de habitación, a $750 \mathrm{~m}$ Norte de la casa de su mamá por lo que ellas se dirigen para darle la bienvenida. Considerando como punto de referencia la casa de Carolina: a. Determine su ubicación actual en metros. b. Determine la distancia en metros. (MEP, 2012, p. 282)

Otro ejemplo de problema contiene información sobre animales que habitan fuera de Costa Rica, por lo que la población local no está familiarizada con este contexto.

El yak es un animal que habita en las montañas del Tíbet a unos $5000 \mathrm{~m}$ sobre el nivel del mar y el cachalote vive $5900 \mathrm{~m}$ más abajo. Determine la altura en la que suele vivir este último. (MEP, 2012, p. 280)

\subsection{Resultados sobre la contextualización de los problemas}

En los fundamentos teóricos de los Programas de Estudio de Matemáticas del MEP (2012) se reitera la importancia de trabajar problemas asociados a entornos reales, físicos, sociales y culturales (p. 13) y se plantea "una contextualización activa que estimule la acción estudiantil, lo que requiere el uso importante de modelos sobre la realidad cercana" (p. 36). De lo anterior, radica la importancia de analizar si los problemas que se presentan poseen una contextualización activa o si, por el contrario, presentan una contextualización artificial. Para clasificar el problema según una contextualización activa, se debe preguntar si el contexto es necesario para resolver el problema, según lo que establecen el MEP (2012) y Ruiz (2017). En el caso de que la respuesta sea negativa, se trata de una contextualización artificial o forzada.

Una vez descartados los de contexto matemático/científico, 57 problemas fueron analizados según la contextualización activa o artificial. De estos, 45 presentan una contextualización activa y 12 una contextualización artificial. Es decir, poco más del 
$21 \%$ de los problemas tiene un contexto forzado, que no es necesario para dar significado a la situación planteada, ni para brindar solución a este.

Los problemas de las Figuras 3 y 4 plantean un contexto necesario para su resolución, por lo que hay contextualización activa. La información de la Isla del Coco (forma y dimensiones a consultar en un mapa) es imprescindible para aproximar su área. Por su lado, el contexto es necesario para resolver el problema donde se requiere saber la relación entre las dimensiones de la habitación y de las losetas, de modo que el aprendiz aplique el algoritmo de la división.

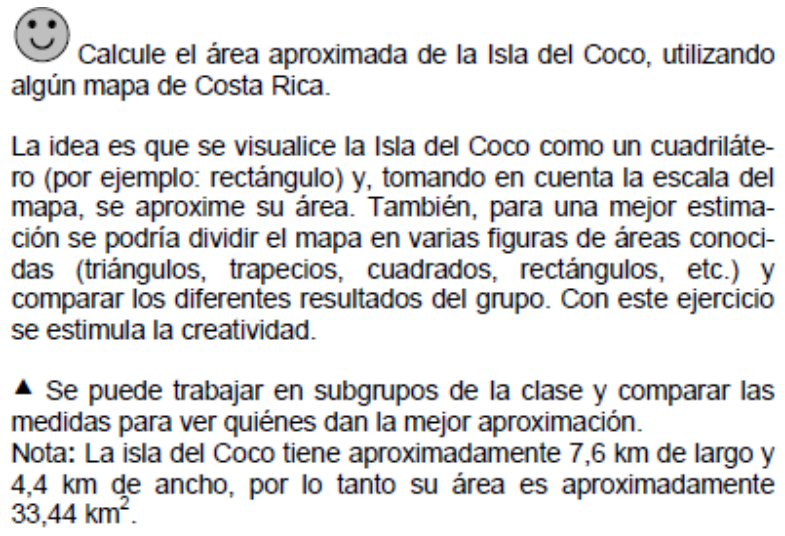
ro (por ejemplo: rectángulo) y, tomando en cuenta la escala del mapa, se aproxime su área. También, para una mejor estimación se podría dividir el mapa en varias figuras de áreas conocidas (triángulos, trapecios, cuadrados, rectángulos, etc.) y comparar los diferentes resultados del grupo. Con este ejercicio se estimula la creatividad.

- Se puede trabajar en subgrupos de la clase y comparar las medidas para ver quiénes dan la mejor aproximación.

Nota: La isla del Coco tiene aproximadamente $7,6 \mathrm{~km}$ de largo y $4,4 \mathrm{~km}$ de ancho, por lo tanto su área es aproximadamente $33,44 \mathrm{~km}^{2}$.

Figura 3. Problema con contextualización activa. Fuente: MEP (2012, p. 305)

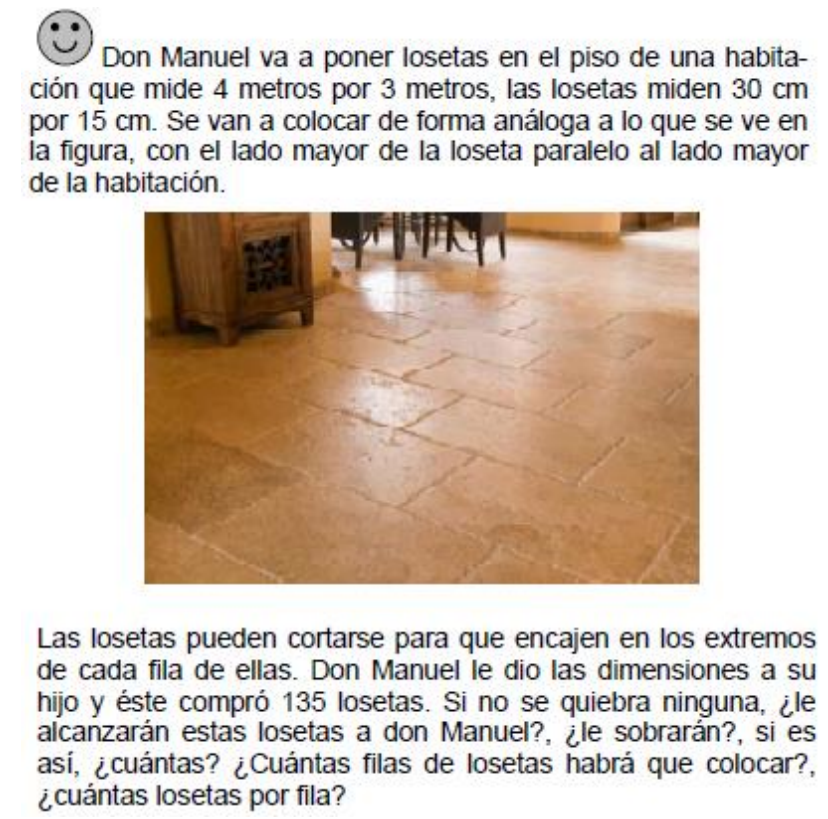

Figura 4. Problema con contextualización activa. Fuente: MEP (2012, p. 277)

Los problemas con contextualización artificial son aquellos que presentan datos descriptivos (relacionados con otras ciencias o datos geográficos), que no son necesarios para su resolución. Si se eliminan estos datos descriptivos, el problema podría presentarse con un contexto matemático. El siguiente problema es un ejemplo:

El monte Everest (la montaña más alta del mundo) es 5.413 metros más alto que el volcán Irazú (uno de los puntos más altos de Costa Rica). Si la suma de sus alturas es 12.283 metros, plantee una ecuación que permita calcular la altura de cada uno de ellos. (MEP, 2012, p. 335)

Aquí se debe responder a la habilidad de plantear y resolver problemas en contextos reales. Se aprecia que el contexto se limita a indicar que hay una cantidad conocida 
(5.413) y una incógnita, cuya suma es 12.283 . Se podría prescindir del contexto y quedar redactado así: la suma de dos números es 12.283 y uno de ellos es 5.413 , ¿cuál es el otro número? Por tanto, la contextualización es artificial.

Otra indicación puntual pone en relieve una posible conexión entre las ecuaciones lineales, el arte y la historia, al tiempo que "confirma la utilidad de las matemáticas en diversos ámbitos de la vida" (MEP, 2012, p. 336). Sin embargo, los datos históricos y artísticos no son esenciales para resolver el problema.

Una pintura muy famosa es la Gioconda del artista Leonardo da Vinci. Esta pintura se encuentra en el Museo de Louvre en París, Francia. El cuadro tiene forma rectangular y su altura es 24 centímetros más que su ancho. El perímetro del cuadro es de 260 centímetros. Calcule la altura y el ancho del cuadro. (MEP, 2012, p. 336)

La contextualización del problema anterior es artificial, puesto que es un problema matemático con detalles contextuales innecesarios para su resolución. Este podría resolverse con una redacción del tipo: determine la altura y la base de un rectángulo cuya altura es $24 \mathrm{~cm}$ mayor que la longitud de su base.

Un problema posee contextualización significativa para participantes de un grupo, si se puede presentar la situación en su vivencia diaria o laboral y si la pregunta es conforme a una problemática que podría enfrentarse en la realidad. De los 45 problemas clasificados con contextualización activa, 6 presentan contextualización significativa y los otros $39(86,6 \%)$ contextualización no significativa. Para decidir los problemas con contextualización significativa, analizamos si se presentarían y resolverían de esa forma en la realidad del grupo involucrado. Para estos problemas se espera que el aprendiz realice cierto trabajo de matematización o modelización (Niss, 1995). La Tabla 1 resume los problemas con contextualización significativa hallados.

Tabla 1. Problemas con contextualización significativa

\begin{tabular}{|c|c|c|}
\hline Conocimiento & Sujeto & Resumen del problema \\
\hline $\begin{array}{l}\text { Operaciones } \\
\text { combinadas }\end{array}$ & Compradora & $\begin{array}{l}\text { Una joven va a la feria del agricultor y realiza diversos cálculos } \\
\text { para conocer el total que debe pagar. }\end{array}$ \\
\hline $\begin{array}{l}\text { Orden de } \\
\text { números enteros }\end{array}$ & Empresarios & $\begin{array}{l}\text { A través de una gráfica de ganancias y pérdidas de una empresa, } \\
\text { se deben establecer comparaciones. }\end{array}$ \\
\hline $\begin{array}{l}\text { Razones } \\
\text { trigonométricas }\end{array}$ & Albañil & $\begin{array}{l}\text { Se desea conocer la medida de una rampa, para que cumpla con } \\
\text { la Ley } 7600 \text {, para accesibilidad sin exclusión. }\end{array}$ \\
\hline $\begin{array}{l}\text { Ecuación de } \\
\text { una recta }\end{array}$ & Emprendedor & $\begin{array}{l}\text { Un emprendedor busca el precio de venta de un producto } \\
\text { sabiendo costos de producción por unidad e inversión inicial. }\end{array}$ \\
\hline $\begin{array}{l}\text { Medidas de } \\
\text { posición }\end{array}$ & Estudiante & $\begin{array}{l}\text { Un estudiante tiene las notas de los rubros que le calificaron en } \\
\text { un curso y necesita su promedio ponderado. }\end{array}$ \\
\hline $\begin{array}{l}\text { Muestras } \\
\text { aleatorias }\end{array}$ & $\begin{array}{l}\text { Organizadores de } \\
\text { programa de salud }\end{array}$ & $\begin{array}{l}\text { Una organización necesita escoger aleatoriamente a } 15 \\
\text { estudiantes de noveno nivel para un programa de salud. }\end{array}$ \\
\hline
\end{tabular}

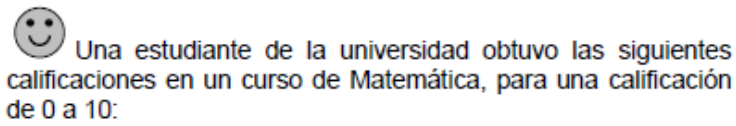
de 0 a 10

\begin{tabular}{|l|c|}
\hline \multicolumn{1}{|c|}{ Pruebas } & Calificaciones \\
\hline Primer examen corto & 6,00 \\
\hline Segundo examen corto & 5,50 \\
\hline Tercer examen corto & 6,50 \\
\hline Proyecto & 6,00 \\
\hline Primer parcial & 7,50 \\
\hline Segundo parcial & 8,50 \\
\hline
\end{tabular}

a. Los exámenes cortos tenían un valor de $5 \%$ cada uno, el proyecto valía $15 \%$ y los exámenes parciales $35 \%$ cada uno. Si la nota mínima de aprobación es un 7,00, ¿la estudiante aprobó el curso?

Figura 5. Problema con contextualización significativa. Fuente: MEP (2012, p. 434) 
La Figura 5 ilustra un problema con contextualización significativa. Un aprendiz puede encontrarse en una situación como la que describe el problema. Aunque las calificaciones están dadas de 0 a 10 y no de 0 a 100 como en secundaria, en varias universidades de Costa Rica, sí se usa esta escala. Para estos análisis, es importante ubicarse en la posición del sujeto del problema, con el fin de constatar si efectivamente este usará un método matemático como el que se sugiere; de lo contrario, se trata de una contextualización no significativa. Por ejemplo, la Figura 6 presenta una situación que, si bien puede ser conocida (un balneario), plantea una problemática que en el contexto real no interesaría. Un bañista no calcularía, de la manera que se propone en el enunciado, la cantidad de centímetros que debe bajar antes de que el agua le cubra los hombros, máxime que las medidas que se proponen no están disponibles en una piscina.
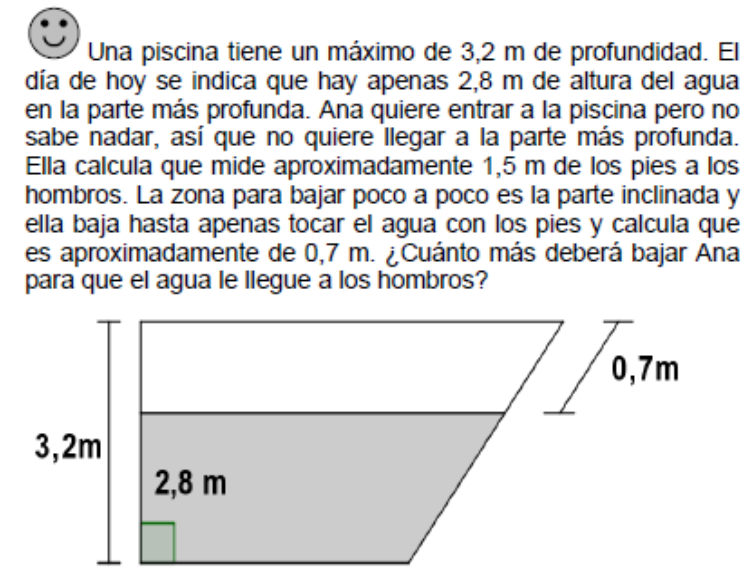

Figura 6. Problema con contextualización no significativa. Fuente: MEP (2012, p. 313)

Otro problema con contextualización no significativa se ejemplifica en el siguiente fragmento, donde se plantea comprar una escalera para subir al techo de su casa.

Diego necesita comprar una escalera para subirse al techo de su casa. El techo está a una altura de 97 pulgadas. Para poder tener una buena estabilidad en la escalera al apoyarse en la pared, las patas de la escalera deben estar a una distancia de entre 30 y 40 pulgadas.

¿Cuál podría ser la medida aproximada de la escalera? (MEP, 2012, p. 315)

Según se escribe en la habilidad asociada, la idea es introducir el teorema de Pitágoras. La situación descrita tiene un contexto real; no obstante, en la cotidianeidad, no se utilizaría dicho teorema. La estimación sería posiblemente una estrategia más adecuada. Además, rara vez se compra una escalera para usarla solo en una situación. Otro aspecto es que la unidad de medida no es del Sistema Internacional de Unidades de Medida (donde el metro es la unidad de longitud), el utilizado en Costa Rica.

En otro problema, se solicita representar tabularmente una función lineal que relacione el número de kilómetros recorridos por un taxi, con la tarifa a pagar del servicio, sabiendo que el primer kilómetro tiene un costo de $\mathbb{C} 550$ y cada kilómetro adicional vale $\mathbb{C} 200$ (MEP, 2012, p. 331). En Costa Rica para calcular una tarifa, además del kilometraje, se considera el tiempo que se tarda en el servicio. Debido a la complejidad de las mediciones, los taxistas usan un taxímetro conocido como "María", que efectúa dicho cálculo. Ni el chofer ni el usuario hacen esos cálculos de manera manual. Por tanto, este problema también es de contextualización no significativa.

Hemos detectado cierta escasez de problemas con contextualización significativa en los Programas de Estudio de Matemáticas del MEP (2012), donde se explica que "al usar o aplicar las matemáticas dentro de contextos reales (bien seleccionados) se 
promueve el contacto con los objetos matemáticos en su relación privilegiada con la realidad de donde emergieron" (p. 28). La Figura 7 presenta un resumen de resultados.

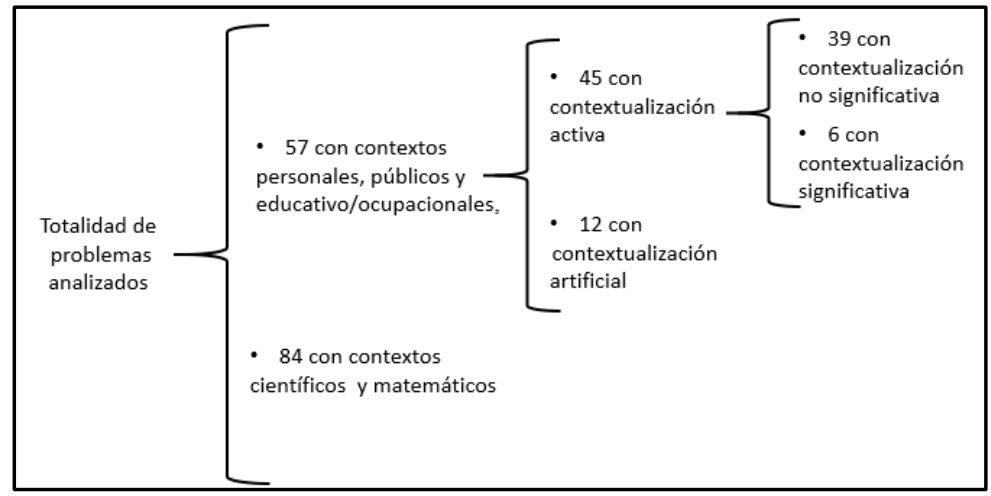

Figura 7. Clasificación de problemas en valores absolutos según contexto-contextualización

\section{Conclusiones}

Desde hace tiempo la investigación en educación matemática ha establecido la importancia de tener en cuenta aspectos culturales en la comprensión y el diseño de los procesos de enseñanza y aprendizaje (Bishop, 1991; Presmeg, 2007). Estos esfuerzos se concretan con el uso de contextos de la vida cotidiana para enseñar matemáticas. En esta línea de atención a lo cultural, los fundamentos teóricos del actual currículo de matemáticas de Costa Rica se promueve la enseñanza con situaciones cercanas para el aprendiz, potenciando el uso de contextos reales y la modelización.

Al analizar los problemas en los Programas de Estudio de Matemáticas del MEP (2012), para el III y IV ciclo y profundizando sobre la contextualización activa que se propone en los fundamentos teóricos de este documento, identificamos sin embargo algunos desajustes. Mientras que en el currículo se explica que el aprendizaje no debe limitarse al dominio de técnicas sofisticadas o estructuras abstractas alejadas del entorno, más del 59,5\% de los problemas pertenecen a contextos matemáticos o científicos que no consideran realidades cercanas. En los fundamentos teóricos del programa, además, se explica la necesidad de impulsar unas matemáticas para todos, pero los ejemplos no contemplan problemas que respondan a la realidad de habitantes de zonas rurales ni indígenas. Si bien es cierto que se deben hacer las adaptaciones necesarias, según el entorno de enseñanza, brindar problemas con contextos no urbanos enriquecería los Programas de Estudio de Matemáticas en Costa Rica.

En cuanto a los problemas con contextualización significativa, en los análisis hemos evidenciado que este tipo de contextualización es mínima; en su lugar, hay muchos problemas que se alejan como se abordarían en la realidad. Algunos problemas, con pequeñas modificaciones, pueden transformarse para que la contextualización resulte significativa, lo que propiciaría una vivencia más cercana de las matemáticas del entorno. En otros es necesaria la construcción de problemas nuevos que respondan a la habilidad por desarrollar, tal como esta se usaría en una situación real. En este sentido, resulta conveniente incentivar investigaciones que utilicen como base los contextos de los problemas propuestos por el MEP (2012) con el fin de mejorar aquellos con potencial para mostrar de manera más natural las matemáticas como una actividad humana y social, tal como lo expresan Freudenthal (2002), Niss (1995) y D’Ambrosio (2008).

Nuestra investigación tiene la limitación de no informar respecto a cómo las indicaciones del currículo se llevan a la práctica en las aulas, además de centrarse en las edades escolares de secundaria. Se podría entonces completar el estudio con 
observaciones de aulas en distintas zonas del país y ver cómo el profesorado reinterpreta las indicaciones curriculares en su práctica docente y con respecto a más edades escolares. En cualquier modo, creemos que la actual investigación aporta a la didáctica de las matemáticas un sistema de subcategorías para contexto y contextualización que permite estudiar el currículo material intencionado, a la vez que puede servir como guía para analizar problemas en otros documentos tales como libros de textos y unidades didácticas. Las categorías y subcategorías propuestas también proporcionan indicadores de apoyo a la selección y producción de problemas matemáticos que se articulen mediante una contextualización activa y significativa.

\section{Agradecimientos}

Proyecto PID2019-105601GB-I00, Ministerio de Ciencias e Innovación de España, Proyecto 0408-19, Universidad Nacional de Costa Rica.

\section{Referencias}

Albanese, V., Adamuz-Povedano, N., \& Bracho-López, R. (2017). Development and contextualization of tasks from an ethnomathematical perspective. En A. Chronaki (Ed.), Mathematics education and life at times of crisis (pp. 205-211). Volos, Grecia: Thessaly University.

Aroca, A. (2013). Los escenarios de exploración en el Programa de Investigación en Etnomatemáticas. Educación Matemática, 25(1), 111-131.

Bardin, L. (2012). Análisis de contenido. Madrid: Akal Universitaria.

Bishop, A. J. (1991). Mathematical enculturation: A cultural perspective on mathematics education. Dordrecht, Holanda: Kluwer / Springer.

Bishop, A. J. (2005). Aproximación sociocultural a la educación matemática. Cali, Colombia: Universidad del Valle, Instituto de Educación y Pedagogía.

Blanco, L. J., \& Pino, J. (2016). ¿Qué entendemos por problema de matemática? En L. J. Blanco, J. Cárdenas, \& A. Caballero (Eds.), La resolución de problemas de matemáticas en la formación inicial de profesores de primaria (pp. 81-92). Cáceres: Universidad de Extremadura.

Cabrera, F. C. (2005). Categorización y triangulación como procesos de validación del conocimiento en investigación cualitativa. Theoria, 14(1), 61-71.

D’Ambrosio, U. (2008). Etnomatemática. Eslabón entre las tradiciones y la modernidad. México D. F.: Limusa.

Freudenthal, H. (2002). Revisiting mathematics education. China lectures. New York: Kluwer / Springer.

Fuentes, C. (2013). Etnomatemática y escuela: Algunos lineamientos para su integración. Revista Científica, n. especial, 46-50.

Gutstein, E., Lipman, P., Hernandez, P., \& De los Reyes, R. (1997). Culturally relevant mathematics teaching in a Mexican American context. Journal for Research in Mathematics Education, 28(6), 709-737.

Instituto Nacional de Estadística y Censo (2012). X Censo Nacional de Población y VI de Vivienda Resultados Generales. San José, Costa Rica: Autor. 
MEP (2012). Programas de Estudio de Matemáticas. Educación General Básica y Ciclo Diversificado. San José: Ministerio de Educación Pública de Costa Rica.

Niss, M. (1995). Las matemáticas en la sociedad. UNO-Revista de Didáctica de las Matemáticas, 6, 45-58.

Nuñez, J., \& Font, V. (1995). Aspectos ideológicos en la contextualización de las matemáticas: una aproximación histórica. Revista de Educación, 506, 293-314.

OECD (2004). Learning for tomorrow's world: First results from PISA 2003. París: Organización para la Cooperación y el Desarrollo Económico.

Palm, T. (2008). Impact of authenticity on sense making in word problem solving. Educational Studies in Mathematics, 67(1), 37-58.

Peña, P. (2014). Etnomatemáticas y currículo: Una relación necesaria. Revista Latinoamericana de Etnomatemática, 7(2), 170-180.

Planas, N. (2015) (Ed.). Avances y realidades de la educación matemática. Barcelona: Colección Crítica y Fundamentos, Graó.

Planas, N., \& Alsina, Á. (2009) (Eds.). Educación matemática y buenas prácticas. Educación infantil, primaria, secundaria y educación superior. Barcelona: Graó.

Presmeg, N. (2007). The role of culture in teaching and learning mathematics. En F. K. L. Jr (Ed.), Second handbook of research on mathematics teaching and learning (pp. 435-458). Charlote, NC: Information Age Publishing.

Puig, A. (1955). Decálogo de la Didáctica Matemática Media. Gaceta Matemática, 7(5), 130-135.

Ramos, A. B., \& Font, V. (2006). Contesto e contestualizzazione nell'insegnamento e nell'apprendimento della matematica. Una prospettiva ontosemiotica. La Matematica e la sua Didattica, 20(4), 535-556.

Rico, L. (2006). La competencia matemática en PISA. PNA, 1(2), 47-66.

Ruiz, Á. (2000). El desafío de las matemáticas. San José, Costa Rica: Editorial Universidad Nacional.

Ruiz, Á. (2013) La educación matemática en Costa Rica: Antes de la reforma. Cuadernos de Investigación y Formación en Educación Matemática, 8(n. especial), $10-16$.

Ruiz, Á. (2017). Los contextos en el currículo de Matemáticas de Costa Rica. Cuadernos de Investigación y Formación en Educación Matemática, 12(n. especial), 72-76.

\section{Referencias de los autores}

Gilberto Chavarría-Arroyo, Universidad Nacional (Costa Rica).

gilberto.chavarria.arroyo@una.cr

Veronica Albanese, Universidad de Granada (España).vealbanese@ugr.es 


\title{
Mathematical problems in the case of a curriculum: Analysis based on context and on contextualization
}

\author{
Gilberto Chavarría-Arroyo, Universidad Nacional \\ Veronica Albanese, Universidad de Granada
}

In 2012, an educational reform in Costa Rica introduces a new mathematical curriculum for schools, with the resolution of contextualized (real life) problems as one of its basis The aim of the research presented in the article is to analyze the contexts and contextualization of problems proposed in this curriculum in order to verify whether contexts are somehow linked to the lives of learners and their contextualizations are active -i.e. needed in the understanding and resolution of the problems- and significant -i.e. authentic in the sense of similar in the situation proposed but also in the resolution with respect to what would be likely done in the out-of-school practice-. In particular, we take the problems proposed from $7^{\text {th }}$ to $12^{\text {th }}$ grades. Our literature review allows us to construct a system of two major categories, those of context and contextualization, that are then developed into subcategories. A qualitative content analysis of this net of categories and subcategories is followed by a quantitative recount. Amongst some of the main results, we find that more than half $(59.5 \%)$ of the 141 problems analyzed are of the subcategory called scientific-mathematical context, while any of them belong to the subcategories called rural and indigenous contexts. Although some of the problems refer to Costa Rica, in a few of them the situation described cannot be interpreted the subcategory called Costa Rican context. In addition, and in disconnection with the theoretical foundations of the case of the curriculum considered, there are some problems whose contextualization is not the subcategory called active, that is, whose contexts are not relevant in either the formulation or the resolution. Only a minority of the problems analyzed fall into a contextualization of the subcategory called significant, that is, whose contextualization reflects what could be reasonably seen in the practices of the groups suggested in the wording. Together with these evidences, our study also offers supportive tools and exemplifications for the selection and construction of problems with both active and significant contextualizations. At this stage of our research, the overall conclusion is that the notions of context and contextualization are highly valuable for the improvement of school mathematics curricula oriented to work with mathematical problems and, at the same time, their interpretation in practice is not trivial since a diversity of challenges emerge from the attention to social and cultural issues in mathematics teaching and learning. More research is thus necessary. 